\title{
Clinical practice guidelines in multisystem inflammatory syndrome (MIS-C) related to COVID-19: a critical review and recommendations
}

\author{
Alanood S. Algarni ${ }^{1}$ - Njoud M. Alamri ${ }^{2} \cdot$ Noor Z. Khayat $^{2} \cdot$ Raghad A. Alabdali $^{2} \cdot$ Rawabi S. Alsubhi $^{2} \cdot$ \\ Shahad H. Alghamdi
}

Received: 21 August 2021 / Accepted: 5 December 2021 / Published online: 4 January 2022

(c) Children's Hospital, Zhejiang University School of Medicine 2022

\begin{abstract}
Background Multisystem inflammatory syndrome in children (MIS-C) is a serious health condition that develops from and is linked to coronavirus disease 2019. MIS-C is considered a multi-organ dysfunction involving cardiac, renal, respiratory, hematologic, gastrointestinal and neurological symptoms and groups of signs and symptoms such as rash or bilateral nonpurulent conjunctivitis, hypotension or shock and acute gastrointestinal problems, which require immediate therapeutic intervention to prevent the aggravation of the patient's health condition. MIS-C is relatively new in the field of evidence-based medicine; however, there are several clinical guidelines for good clinical practice. For every disorder, the guidelines have different suggestions. Hence, based on the current status of the evidence, recommendations have been combined to form a unified guideline for therapeutic management.

Methods This paper compares and evaluates the current MIS-C-specific clinical practice guidelines (namely, American Academy of Pediatrics, American College of Rheumatology, Helen DeVos Children's Hospital Foundation, Children's Hospital of The King's Daughters, and the Infectious Diseases Society of America). The compiled literature was then assessed by the authors separately, and an algorithm was proposed for each disorder, taking into consideration the various guidelines proposed for the management of the disorder.

Results The features of MIS-C patients are unified; this is very helpful in managing its symptoms and decreasing mortality rates. In addition, recommendations for pharmacological treatment for MIS-C symptoms are formulated after crosscomparison across five different guidelines.

Conclusions This study provides a general interpretation of the results in the context of other evidence and implications for future research. It proposes a unified guideline based on the current evidence, with the best potential to maintain suitable clinical standards in the Saudi Arabian Ministry of Health.
\end{abstract}

Keywords Coronavirus disease $2019 \cdot$ Multisystem inflammatory syndrome $\cdot$ Pediatric multisystem inflammatory syndrome

\section{Introduction}

The pneumonia cases caused by the novel $\beta$-RNA coronavirus were named by the World Health Organization (WHO) coronavirus disease 2019 (COVID-19) [1]. Early in 2020,

Alanood S. Algarni

aagarni@uqu.edu.sa

1 Department of Pharmacology and Toxicology, Faculty of Pharmacy, Pharmacy Collage, Umm Al-Qura University, Makkah 21955, Saudi Arabia

2 Pharmacy Collage, Umm Al-Qura University, Makkah, Saudi Arabia the United Kingdom National Health Service observed a nationwide increase in a rare but serious health condition in children called multisystem inflammatory syndrome in children (MIS-C), which developed from and was linked to COVID-19 [2-4]. On April 7, 2020, the first MIS-C case of a 6-month-old infant in the USA was diagnosed with Kawasaki disease $(\mathrm{KD})$ due to the similarities in the clinical features of MIS-C and KD [2-4]. A recent report by Riphagen et al. studied the clinical features of a group of children who had antibodies for severe acute respiratory syndrome coronavirus 2 (SARS-CoV-2) and had a severe hyperinflammatory shock, which clustered at a rate of one or two children per week [5]. They found that the clinical presentations of these 
cases were closely related to the characteristics of KD and toxic shock syndrome [5]. A further study reported more cases that were broadly distributed and required immediate admission to intensive care units [6]. On May 1st, 2020, the UK Royal College of Paediatrics and Child Health published guidance on MIS-C [2]. Since then, several other worldwide health organizations have reported that MIS-C is temporally associated with COVID-19, with the development of symptoms two to six weeks after COVID-19 incidence [2-8]. However, the association between COVID-19 and MIS-C is not completely understood, which makes healthcare management more complicated. The first MIS-C case in Saudi Arabia of a 13-year-old female child, reported in September 2020, died despite critical care and management based on the Saudi Ministry of Health guidelines for COVID-19 management [7]. Currently, there are no MIS-C-specific clinical practice guidelines established by the Saudi Ministry of Health. According to the latest statistical update by the Centers for Disease Control and Prevention in the USA in early 2021, the total number of MIS-C cases increased to 1659 cases and 26 MIS-C deaths [9-11]. The median age of the patients ranged from seven to ten years, and more than $59 \%$ of all patients were male [8]. Data suggest that race has a strong association with MIS-C [2]. More than 70\% of reported cases have occurred in children who are Hispanic or Latino [9]. The five main features of MIS-C, according to the WHO, are to be $0-19$ years of age with a fever $>3$ days and at least two of the following: rash or bilateral non-purulent conjunctivitis or mucocutaneous inflammation signs, hypotension or shock, features of myocardial dysfunction, pericarditis, valvulitis or coronary abnormalities, evidence of coagulopathy, and acute gastrointestinal problems, as well as elevation of the inflammatory markers with no other obvious microbial cause of inflammation plus either COVID-19 antigen test/serology positive or contact with COVID-19 patients [12].

Because some of the symptoms of MIS-C and KD are similar, it may be hard to tell the difference between them based on symptoms alone. MIS-C development after infection with COVID-19 is significant information to help confirm the diagnosis. Most of the available guidelines for good clinical practice are widely known and accepted, such as the American Academy of Pediatrics (AAP) guideline, updated on 17 November 2020 [1], the American College of Rheumatology (ACR) guideline, submitted to Arthritic Rheumatology on 15 February 2021 [2], and the HDVCH guideline by Spectrum Health Helen DeVos Children's Hospital, located in Grand Rapids, Michigan, United States on 10 January 2020 [3]. Children's Hospitals and Clinics of Minnesota is one of the largest freestanding pediatric health systems in the United States; it published MIS-C guidelines in January
2020 [4]. The pediatric intensive care unit (PICU) guidelines issued by Nature Research (which has principal offices around the world in London, New York, Berlin, Shanghai and Tokyo) were published online on 7 July 2020 [5]. The last CHKD (Children's Hospital of The King's Daughters) guideline was published on 31 May 2020) [6].

Many pharmacological therapies are used to manage the symptoms of MIS-C disease according to various guidelines available worldwide, as mentioned above $[6,7]$. All the available clinical practice guidelines recommend the use of certain medications considered essential in managing MIS-C symptoms, including intravenous immunoglobulin (IVIG) at a dose of $2 \mathrm{~g} / \mathrm{kg} / \mathrm{dose}$ [6, 7]. In addition, due to the multi-inflammatory nature of the disease, therapies that have anti-inflammatory and immunosuppression activity must be used. The previously mentioned guidelines unanimously agree on the use of steroid medications such as methylprednisolone for this purpose, as well as biologics such as anakinra and remicade or interleukin-6 inhibitors. With follow-up, depending on clotting factors and risk factors, the use of low-dose aspirin as prophylaxis has been recommended, especially for patients who have developed KD-like features. For community-acquired shock, some guidelines such as AAP and Children's Hospitals and Clinics of Minnesota have recommended the use of antibiotics such as vancomycin, ceftriaxone and clindamycin along with other treatments $[1,5]$.

As in any other pediatric condition, clinicians need to follow the most reliable guidelines. MIS-C is assumed to be a critical condition with multiple serious complications that vary from patient to patient. Thus, clinicians must follow the safest and most effective treatment guidelines to minimize mortality and reduce the risk of long-term sequelae. Treatment for MIS-C aims to decrease systemic inflammation and restore organ function. Regarding the epidemiology, pathogenesis, clinical scope and long-term effects of MIS$\mathrm{C}$, much remains unknown.

Analysis of symptom, risk of death and quality of life based on each guideline and previous analysis to adopt the most accurate guideline with minimum harmful effect; this will help to decrease the risk of complications and mortality rate. As should be expected, all the above-mentioned guidelines need to be uniform and precise. However, as this review critique shows, there are several differences when these guidelines are compared. This paper, therefore, attempts to compare the treatment recommendations by the above-mentioned guidelines for MIS-C multi-symptoms and disorders and evaluates the differences among them. It also suggests a unified guideline, putting together common recommendations and available evidence from all the above. 


\section{Methods}

A comprehensive literature search for published MIS-C practice management guidelines was conducted through many sources, such as ProQuest, Scopus, ScienceDirect, Wiley, Saudi Digital Library and PubMed. Google Scholar was used for additional relevant studies from 2020 to February 2021 . The keyword searches were related to the main subject (e.g., "MIS-C or multisystem inflammatory syndrome", "PMIS or pediatric multisystem inflammatory syndrome", "COVID-19") and the management domain (e.g., "antibiotic", "antiviral", "IVIG or immunoglobulin", "anticoagulant or heparin", "antiplatelet or aspirin", "biologic or anakinra or tocilizumab"). The most common guidelines, namely, the APA, ACR, HDVCH, Children Minnesota, PICU, CHKD and Infectious Diseases Society of America (IDSA), were also reviewed. The literature was then assessed by the authors separately and an algorithm was proposed for each disorder, taking into consideration the various guidelines proposed for the management of the specific disorder.

\section{Results}

\section{Definition of multisystem inflammatory syndrome in children according to each guideline (Table 1)}

Various guidelines show minor differences of opinion here, and hence the unified guideline recommending mostly the same criteria to define MIS-C cases.

\section{Age}

All the guidelines and the unified guideline recommend an age $<21$ years. According to case series in the US and UK in 2020, the median age was $8-11$ years (range 1-20 years). There have been rare reports of MIS-C disorder occurring in adults aged in the mid-20s where the patient met the other criteria, with the exception of age [14].

\section{Fever}

There are no differences of opinion among the guidelines in defining the fever, so the unified guideline is the best recommendation: the documented fever $\geq 38.0{ }^{\circ} \mathrm{C}$ $\left(100.4{ }^{\circ} \mathrm{F}\right)$ for $\geq 24$ hours or a report of subjective fever lasting $\geq 24$ hours.

\section{Laboratory evidence of inflammation}

Several differences are apparent in the identification of laboratory markers of inflammation among the various guidelines. The unified guideline recommends laboratory abnormalities noted in all guidelines based on the most frequent markers in critical case series [15-19]. All guidelines considered abnormal blood cell counts, including lymphocytopenia and thrombocytopenia; this is also supported by the unified guideline. In addition, almost all the guidelines recommend measuring inflammatory markers [C-reactive protein (CRP), erythrocyte sedimentation rate (ESR), procalcitonin and ferritin] at the time of admission and then serially to monitor progression. With the exception of the HDVCH and CHKD guidelines, all others recommend the use of both CRP and ESR as routine inflammatory markers. The HDVCH and CHKD guidelines only recommend the use of CRP. Based on recent evidence, the ESR is not useful for serial monitoring because most patients with MIS-C are treated with IVIG, which can elevate the ESR. As in APA, HDVCH and CHKD, the unified guideline recommends monitoring of cardiac markers (troponin and brain natriuretic peptide). In contrast, the ACR and IDSA guidelines offer no specific recommendations for cardiac marker monitoring.

\section{Multisystem involvement and Kawasaki disease-like symptoms}

All the guidelines and the unified guideline recommend the involvement of two or more organ systems, KD-like symptoms and the development of MIS-C after COVID-19 infection by $4-6$ weeks as required criteria to define MIS-C.

\section{Management of multisystem inflammatory syndrome in children according to each guideline (Table 2)}

As the vast majority of evidence supports the use of IVIG, all the guidelines and the unified guideline recommend the continued use of IVIG (1-2 gm/ $/ \mathrm{kg})$ and steroids as an antiinflammatory. According to McArdle et al., in a surveillance study involving patients with MIS-C, primarily treated with IVIG alone, IVIG plus glucocorticoids, or glucocorticoids alone, investigators found that the time until a reduction in disease severity was similar in the three groups [20]. Another study recently reported that initial treatment with a combination of IVIG plus glucocorticoids was associated with a lower risk of cardiovascular dysfunction than IVIG alone [21].

Immunosuppressants (prednisone, azathioprine) suppress the immune system action by curtailing the production of white blood cells (defensive cells), known as T-cells. Therefore, immunosuppressants curtail inflammation and cell damage, thereby minimizing symptoms. For example, the dosage for azathioprine ( $\mathrm{Rx}$ ) is $2-3 \mathrm{mg} / \mathrm{kg}$ once daily for the remission, maintenance and reduction of steroids. The lower dosage may be $100 \mathrm{mg} / \mathrm{day}$ and the length of the 


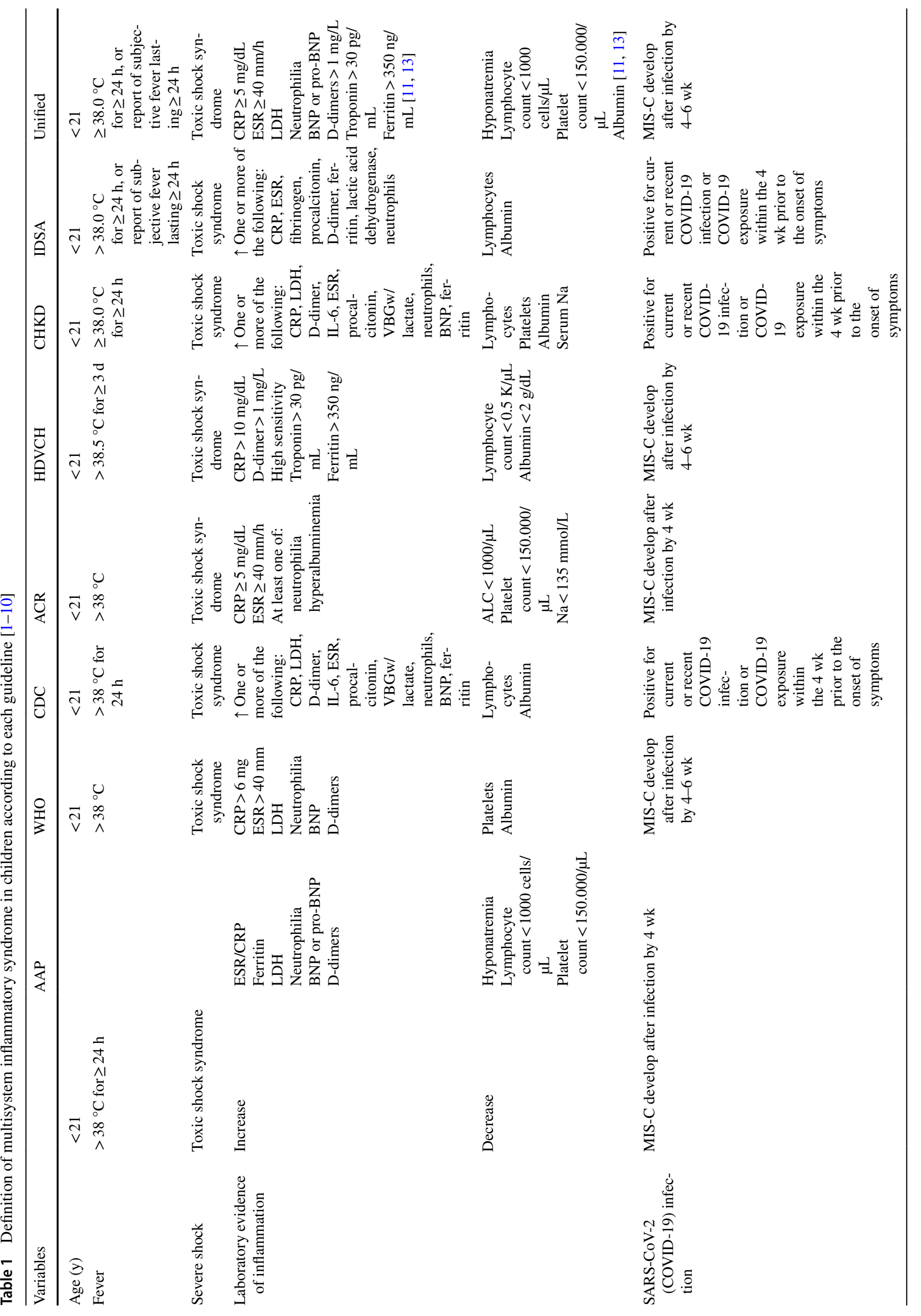




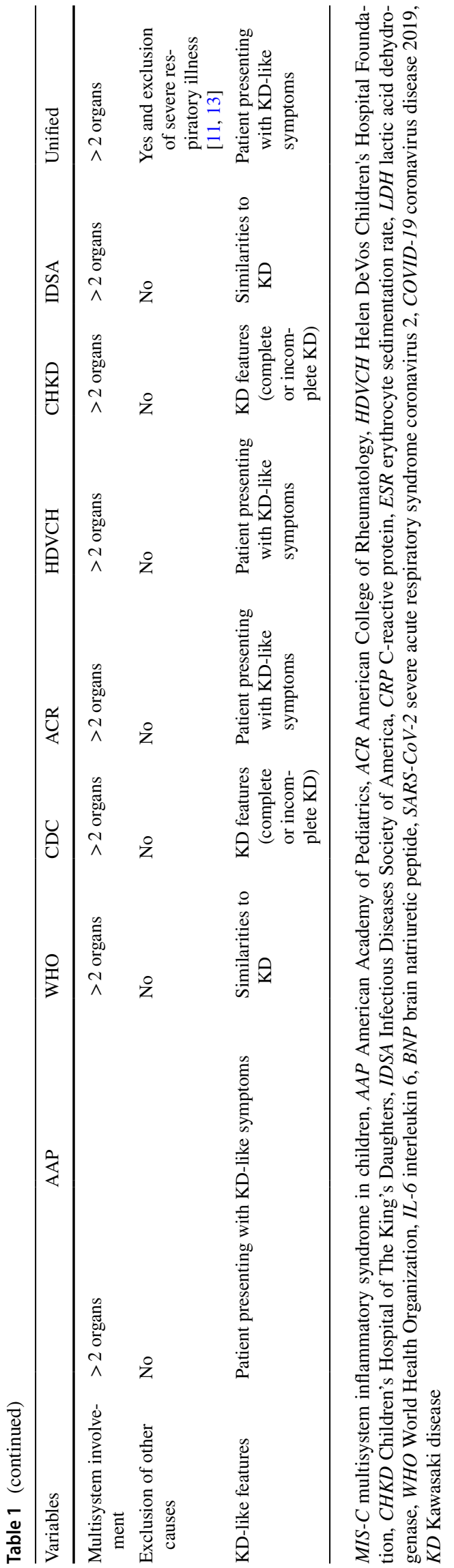

dosage is up to 84 months. Although Schoot et al. asserted that immunosuppressants are typically harmful in the initial COVID-19 treatment phase, the drugs are beneficial in the severe conditions of COVID-19 by repressing the overshooting of the immune responses [22]. At the same time, immunosuppressive indications confirm that they reduce the patient's mechanical ventilation and mortality. Finally, early treatment with methylprednisolone is highly beneficial for a patient's health under severe conditions [22].

According to Al-Ghafry et al., anticoagulants such as warfarin (Rx) or heparin tablets of 3-7 mg, twice a day for three or more months, as the dosages are considered based on the genotypes. The use of anticoagulants requires frequent monitoring until the patient's health condition stabilizes. The primary use of anticoagulants is to curtail clot formation within the arterial and venous of patients with COVID-19. A reduction in blood clots after seven to twelve weeks without prolonged fatigue or any side effects from the patient indicates that the patient's health status is stabilized [23].

Antiplatelet therapy, mainly aspirin, is commonly used in all the guidelines and recent reviews due to concerns about coronary artery involvement and the risk of blood clots [24]; hence, the unified guideline also recommends a low dose. According to Rizk et al., the use of $75 \mathrm{mg}$ (low aspirin dose) for three months decreases cardiovascular diseases and lowers the risks of contracting COVID-19 by 29\% [25]. Merzon et al. confirmed that the test results of COVID-19 patients who used aspirin shifted from positive to negative 3-4 days sooner than those who did not use aspirin, depending on their health conditions. Thus, the findings revealed that a low dosage of aspirin on COVID-19 infection seems to be a promising treatment [26].

Since no separate recommendations exist except for the IDSA guideline, the unified guideline recommends the use of an antiviral, remdesivir in particular, for patients known to have a presentation consistent with typical COVID-19. Only the AAP guideline has recommended antibiotic therapy for patients diagnosed and hospitalized with MIS-C. Recent evidence proposes the initial use of broad-spectrum antibiotics as the symptoms overlap with severe bacterial infections, which is also the recommendation of the unified guideline. With the exception of the CHKD and IDSA guidelines, all other guidelines recommend the use of anticoagulant therapy (enoxapanin) as a standard drug.

\section{Discussion}

Clinical guidelines have been formulated to improve clinical practice, optimize patient healthcare quality and safety and minimize variations in clinical practice [27, 28]. However, it has been shown that clinicians' acceptance and 


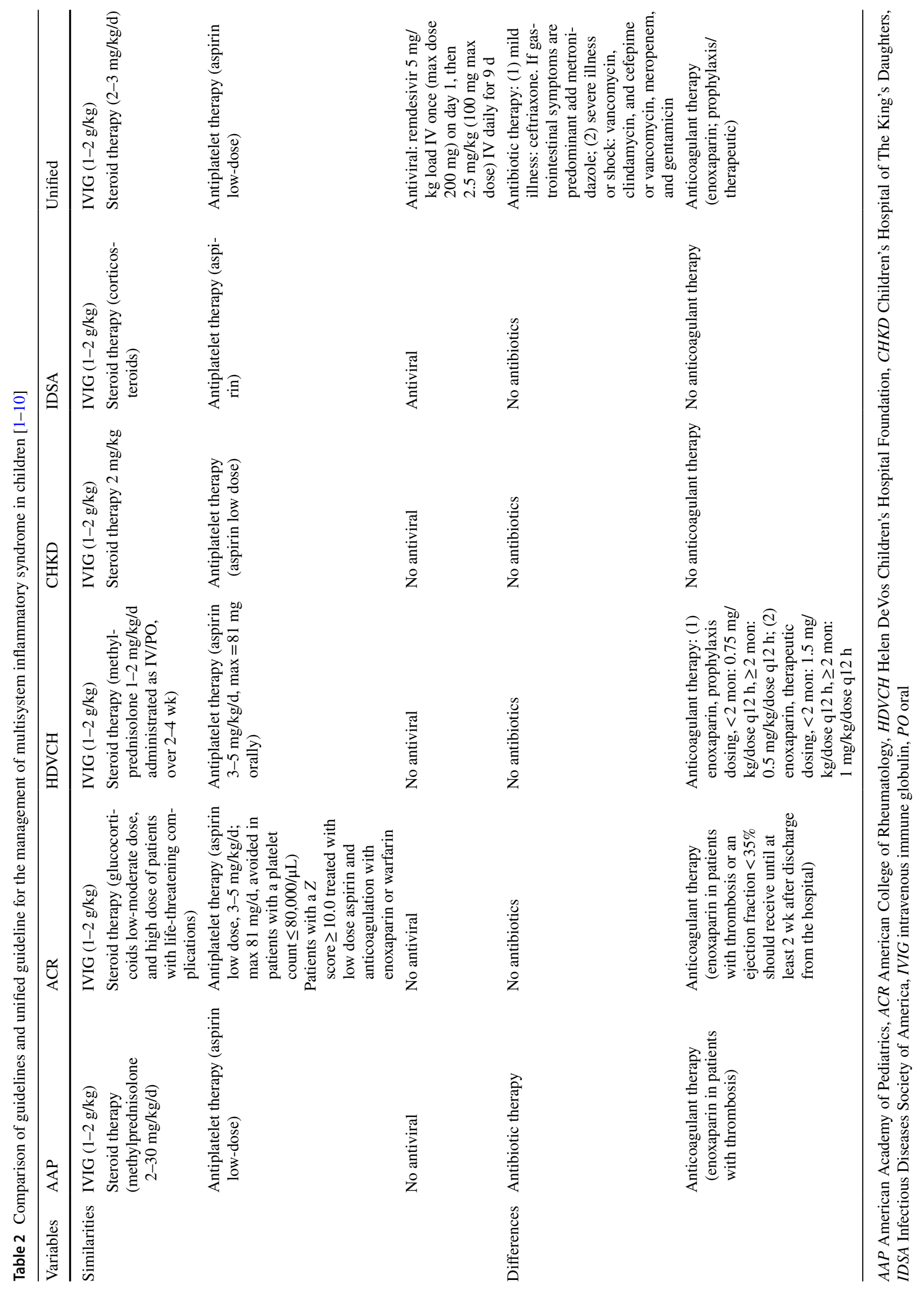


implementation of guidelines are affected by the complexity of translating evidence-based medicine into clinical practice and pharmaceutical sponsorship [27]. In particular, the manipulation of recently established clinical guidelines for critical cases is relatively new to the scope of evidencebased medicine, such as MIS-C. Although there are some differences in the recommendations and evidence levels among the management guidelines established for MIS-C, a comparison of the current management guidelines has, to our knowledge, not been reported.

To improve clinicians' acceptance, an ideal guideline that would be derived from a comprehensive literature review is required. Such a guideline would make recommendations not only for the pharmacological management of children that fulfil the criteria for MIS-C but also for assessment and a multi-disciplinary team approach to interventions to decide the level of care, as the disease manifestations can be critically different. Hence, this study, for the first time, has attempted to comprehensively compare and evaluate the content quality of the five most-followed MIS-C guidelines: applicability and strength of evidence. Then it makes unified guideline recommendations for pharmacotherapy, which may be useful to clinicians and pharmacists.

In contrast to the definition of MIS-C according to each guideline, which was analogous to a fair level of specificity among the compared guidelines, recommendations for treatment were varied. Whereas in some pharmacotherapy fields, recommendations were quite similar among guidelines (e.g., IVIG, steroid therapy, antiplatelet therapy and low-dose aspirin), others differed widely (recommendations on antiviral, antibiotic and anticoagulant therapy). Such variations in recommendations make clinicians' decisions difficult to implement in their daily practice.

The unified guideline evaluates the guidelines' noteworthy recommendations and attempt to establish a broad initial approach to bridge the gap. A unified guideline suggested by Saudi health professionals, with the best potential to maintain the clinical standard, may help clear the confusion stemming from the use of different guidelines around the world. This guideline aims to provide simple, clear protocols for everyone involved in the care of MIS-C patients. Variations in the international guidelines, even simple variations, can affect patient outcomes and increase the risk of deterioration for unknown reasons. Therefore, greater consistency in pharmacotherapeutic recommendations is crucial. This study emphasized the need for continuing oversight of country-specific practices and periodic formal evidence-based inclusion of the guidelines to ensure better care.

McArdle et al. confirmed that the WHO recommendations for perfect criteria for antibody testing by researching SARS-CoV-2 require specificity that can inhibit asymptomatic common infection [20]. The WHO findings reveal that MIS-C typically entails various illnesses for diagnostic test development. Thus, clinicians should be cautious in making treatment decisions during the occurrence of an inflammatory disease caused by SARS-CoV-2 infection. The WHO recommends the use of propensity score weighting to decrease bias by not integrating additional treatments during the primary treatment.

In conclusion, MIS-C is a novel disease for which children with COVID-19 are at high risk. Although there have been an increasing number of case reports and case series, the association between COVID-19 and MIS-C remains unclear. Various guidelines are used for the treatment of MIS-C, such as AAP and IDSA; however, there are no unified guidelines for the treatment of MIS-C. This study compared and evaluated the five most-followed MIS-C guidelines. Based on the management strategies found in the AAP and IDSA guidelines, it is important to know the features of the disease in every patient to determine the appropriate treatment, manage the symptoms and decrease the mortality rate.

Author contributions AAS contributed to study conception, design, supervision, methodology, data curation, and formal analysis. ANM, KNZ, ARA, ARS, and ASH contributed to data collection, analysis, interpretation of results and draft manuscript preparation. All authors reviewed the results and approved the final version of the manuscript.

Funding This research received no specific grant from any funding agency in the public, commercial or profit sectors.

Data availability No datasets were generated or analyzed during the current study.

\section{Declarations}

Ethical approval Not need.

Conflict of interest No financial or non-financial benefits have been received or will be received from any party related directly or indirectly to the subject of this article. The authors have no conflict of interest to declare.

\section{References}

1. American Academy of Pediatrics. Multisystem inflammatory syndrome in children (MIS-C) interim guidance. Retrieved 2 Oct 2020. https://services.aap.org/en/pages/2019-novel-coronaviruscovid-19-infections/clinical-guidance/multisystem-inflammato ry-syndrome-in-children-mis-c-interim-guidance/. Accessed 7 Feb 2021.

2. Henderson LA, Canna SW, Friedman KG, Gorelik M, Lapidus SK, Bassiri H, et al. American college of rheumatology clinical guidance for multisystem inflammatory syndrome in children associated with SARS-CoV-2 and hyperinflammation in pediatric COVID-19: version 2. Arthritis Rheumatol. 2021;73:e13-29. 
3. HDVCH guidelines for management of pediatric patient with multisystem inflammatory syndrome. Spectrum health. https:// www.spectrumhealth.org/for-health-professionals/clinical-pathw ays/management-of-multisystem-inflammatory-syndrome-in-child ren-guideline. Accessed 7 Feb 2021

4. Simon Junior H, Sakano TMS, Rodrigues RM, Eisencraft AP, Carvalho VEL, Schvartsman C, et al. Multisystem inflammatory syndrome associated with COVID-19 from the pediatric emergency physician's point of view. J Pediatr (Rio J). 2021;97:140-59.

5. Riphagen S, Gomez X, Gonzalez-Martinez C, Wilkinson N, Theocharis P. Hyperinflammatory shock in children during COVID-19 pandemic. Lancet. 2020;395:1607-8.

6. Parsons S, Sass L. Children's Hospital of The King's Daughters Health System (CHKDHS). Evaluation and management of COVID-19 multisystem inflammatory syndrome in children (MISC). https://www.chkd.org/uploadedFiles/Documents/COVID-19/ CHKD\%20MIS-C\%20Guideline\%20D2.pdf. Accessed 3 Feb 2021.

7. Al Ameer HH, AlKadhem SM, Busaleh F, AlKhwaitm S, Llaguno MB. Multisystem inflammatory syndrome in children temporally related to COVID-19: a case report from Saudi Arabia. Cureus. 2020;12:e10589.

8. Abrams JY, Godfred-Cato SE, Oster ME, Chow EJ, Koumans EH, Bryant B, et al. Multisystem inflammatory syndrome in children associated with severe acute respiratory syndrome coronavirus 2 : a systematic review. J Pediatr. 2020;226:45-54.e1.

9. Bhimraj A, Morgan RL, Shumaker AH, Lavergne V, Baden L, Cheng VC, et al. Infectious Diseases Society of America guidelines on the treatment and management of patients with COVID19. Clin Infect Dis. 2020. https://doi.org/10.1093/cid/ciaa478.

10. World Health Organization. Multisystem inflammatory syndrome in children and adolescents with COVID-19. www.who.int/publi cations-detail/multisystem-inflammatory-syndrome-in-childrenand-adolescents-with-covid-19. Accessed 5 Feb 2020.

11. Takia L, Angurana SK, Nallasamy K, Bansal A, Muralidharan J. Updated management protocol for multisystem inflammatory syndrome in children (MIS-C). J Trop Pediatr. 2021;67:fmab01.

12. Jiang L, Tang K, Levin M, Irfan O, Morris SK, Wilson K, et al. COVID-19 and multisystem inflammatory syndrome in children and adolescents. Lancet Infect Dis. 2020;20:e276-88.

13. Williams V, Dash N, Suthar R, Mohandoss V, Jaiswal N, Kavitha TK, et al. Clinicolaboratory profile, treatment, intensive care needs, and outcome of pediatric inflammatory multisystem syndrome temporally associated with SARS-CoV-2: a systematic review and meta-analysis. J Pediatr Intensive. 2020. https://doi. org/10.1055/s-0040-1719173.

14. CDC COVID-19 Response Team. Coronavirus disease 2019 in children-United States, February 12-April 2, 2020. MMWR Morb Mortal Wkly Rep. 2020;69:422-6.

15. Brodsky NN, Ramaswamy A, Lucas CL. The mystery of MIS-C post-SARS-CoV-2 infection. Trends Microbiol. 2020;28:956-8.
16. Morris SB, Schwartz NG, Patel P, Abbo L, Beauchamps L, Balan $\mathrm{S}$, et al. Case series of multisystem inflammatory syndrome in adults associated with SARS-CoV-2 infection-United Kingdom and United States, March-August 2020. MMWR Morb Mortal Wkly Rep. 2020;69:1450-6.

17. Verdoni L, Mazza A, Gervasoni A, Martelli L, Ruggeri M, Ciuffreda M, et al. An outbreak of severe Kawasaki-like disease at the Italian epicentre of the SARS-CoV-2 epidemic: an observational cohort study. Lancet. 2020;395:1771-8.

18. Feldstein LR, Rose EB, Horwitz SM, Collins JP, Newhams MM, Son MB, et al. Multisystem inflammatory syndrome in US children and adolescents. N Engl J Med. 2020;383:334-46.

19. Godfred-Cato S, Bryant B, Leung J, Oster ME, Conklin L, Abrams J, et al. COVID-19-associated multisystem inflammatory syndrome in children-United States, March-July 2020. MMWR Morb Mortal Wkly Rep. 2020;69:1074-80.

20. McArdle AJ, Vito O, Patel H, Seaby EG, Shah P, Wilson C, et al. Treatment of multisystem inflammatory syndrome in children. $\mathrm{N}$ Engl J Med. 2021;385:11-22.

21. Son MB, Murray N, Friedman K, Young CC, Newhams MM, Feldstein LR, et al. Multisystem inflammatory syndrome in children-initial therapy and outcomes. N Engl J Med. 2021;385:23-34.

22. Schoot TS, Kerckhoffs AP, Hilbrands LB, Van Marum RJ. Immunosuppressive drugs and COVID-19: a review. Front Pharmacol. 2020;11:1333.

23. Al-Ghafry M, Vagrecha A, Malik M, Levine C, Uster E, Aygun $\mathrm{B}$, et al. Multisystem inflammatory syndrome in children (MIS-C) and the prothrombotic state: coagulation profiles and rotational thromboelastometry in a MIS-C cohort. J Thromb Haemost. 2021;19:1764-70.

24. Panteli D, Legido-Quigley H, Reichebner C, Ollenschläger G, Schäfer C, Busse R. Clinical practice guidelines as a quality strategy. Copenhagen, Denmark: European Observatory on Health Systems and Policies; 2019.

25. Rizk JG, Lavie CJ, Gupta A. Low-dose aspirin for early COVID19: does the early bird catch the worm? Expert Opin Investig Drugs. 2021;30:785-8.

26. Merzon E, Green I, Vinker S, Golan-Cohen A, Gorohovski A, Avramovich E, et al. The use of aspirin for primary prevention of cardiovascular disease is associated with a lower likelihood of COVID-19 infection. FEBS J. 2021;288:5179-89.

27. Samalin L, Guillaume S, Auclair C, Llorca PM. Adherence to guidelines by French psychiatrists in their real world of clinical practice. J Nerv Ment Dis. 2011;199:239-43.

28. Masic I, Miokovic M, Muhamedagic B. Evidence based medicine-new approaches and challenges. Acta Inform Med. 2008;16:219-25.

Publisher's Note Springer Nature remains neutral with regard to jurisdictional claims in published maps and institutional affiliations. 